RECYT

Año 21 / No 32 / 2019 / 87-91

\title{
Descripción morfológica de amebas testadas del género Arcella sp., a través del uso de técnicas multiples
}

\section{Morphological description of testate amoeba of the genus Arcella sp., Through the use of multiple techniques}

\author{
Karen Bracale ${ }^{1, *}$, Roschman-González $^{2}$, Héctor Osorio-Vega ${ }^{1}$ \\ 1- Laboratorio de Patología Celular y Molecular, Centro de Medicina Experimental, Instituto Venezolano de \\ Investigaciones Científicas (IVIC), Km 11 de la Carretera Panamericana, Altos de Pipe, Edo. Miranda - Venezuela. \\ 2- Centro de Microscopía Electrónica "Dr. Mitsuo Ogura", Facultad de Ciencias, \\ Universidad Central de Venezuela (UCV). Caracas - Venezuela. \\ * E-mail: karenbiostar@gmail.com
}

\section{Resumen}

En la investigación se describe morfológicamente amebas del género Arcella sp mediante el uso de técnicas de cultivo, microscopía electrónica de barrido (MEB), y morfometría. Para ello, se tomó una muestra de $5 \mu$ l de estos microorganismos en un medio de cultivo salino, estandarizado previamente. Al tratarse de una muestra acuosa, se realizó una estandarización del método utilizado para observación a través del MEB utilizando una modalidad de bajo vacío o fuerza ambiental. En este sentido, las muestras fueron fijada con $5 \mu$ l de solución Karnovsky al $2 \%$ por 15 min y post-fijada con $5 \mu$ l de solución de tetraóxido de osmio al 1\% por 15 min. Posteriormente, se tomó una muestra de la mezcla, que se colocó en una rejilla de cobre cubierta previamente con una película de nitrocelulosa y una película de carbono para mayor resistencia mecánica de la rejilla, la cual se dejó secar en una estufa a temperatura ambiente por 20 min, una vez colocada la muestra. Finalmente, se observó en un microscopio electrónico de barrido en modalidad de bajo vacío (ESEM) y a partir de las imágenes digitalizadas, se obtuvieron las dimensiones de las características diagnósticas de interés, con la ayuda del programa ImageTool 3.0. Los resultados obtenidos de este estudio permitieron describir de forma general las características diagnósticas de un grupo de amebas testadas pertenecientes al género Arcella sp.

Palabras clave: Amebas; Arcella sp; Morfología; Cultivos; Microscopía electrónica de barrido.

\section{Abstract}

In the research morphologically, amoebas of the genus Arcella sp are described by the use of culture techniques, scanning electron microscopy (SEM), and morphometry. For this, a sample of $5 \mu$ l of these microorganisms was taken in a saline culture medium, previously standardized. Being an aqueous sample, a standardization of the method used for observation through the MEB using a low vacuum mode or environmental force. In this regard, the samples were fixed with $5 \mu$ l of Karnovsky solution at $2 \%$ for 15 min and post-fixed with $5 \mu$ of $1 \%$ osmium tetraoxide solution for $15 \mathrm{~min}$. Subsequently, a sample of the mixture was taken, which was placed in a copper grid previously covered with a nitrocellulose film and coated with a carbon film for greater mechanical strength and allowed to dry in an oven at room temperature for $20 \mathrm{~min}$, once the sample was placed. Finally, it was observed in a scanning electron microscope in low vacuum mode (ESEM) and from the digitized images, the dimensions of the diagnostic characteristics of interest were obtained, with the help of the ImageTool 3.0 program. The results obtained from this study allowed to describe in a general way the diagnostic characteristics of a group of tested amoebas belonging to the genus Arcella sp.

Keywords: Amoebas; Arcella sp; Morphology; Cultures; Scanning electron microscopy.

\section{Introducción}

Las amebas son un grupo amplio y heterogéneo de micro eucariotas que se desplazan a través de proyecciones citoplasmáticas o pseudópodos [1]. Las mismas habitan en suelos húmedos o ambientes acuáticos, desempeñando un papel protagónico en el mantenimiento del flujo de energía y el ciclaje de nutrientes de estos sistemas [2]. Dentro de este grupo, se encuentran las amebas testadas, las cuales presentan una teca calcárea que recubre la célula desnuda. Estas pueden ser encontradas en hábitats acuáticos como lagos, lagunas, ríos, cenotes y aguas subterráneas [3], [4], [5], [6]. 
A nivel morfológico, estas amebas presentan varias características que pueden ser diagnósticas en la identificación de este grupo de microorganismos: la teca rígida, que recubre la membrana de la célula y puede estar constituida por elementos de diversa naturaleza; el pseudostama, el cual es una abertura en la región oral de la teca a través de la cual la ameba interactúa con el medio circundante [7], entre otras características diagnósticas. Estas características son determinantes en la identificación de estos microorganismos y las mismas pueden variar en forma y tamaño, lo que favorece la gran diversidad y heterogeneidad de especies presentes en estos grupos de micro eucariontes [8].

Ahora bien, desde hace ya varias décadas el estudio de estos micro eucariotas de vida libre en sistemas naturales ha tomado auge, ya que se le considera un importante componente de la biota que mantiene los canales de regulación de los sistemas naturales, como la transformación y descomposición de la energía, el mantenimiento de sistemas ecológicos y como bioindicadores de procesos de contaminación de sistemas acuáticos [9], siendo incluso utilizados cotidianamente en actividades de la vida diaria de los humanos, lo que los convierte en un importante grupo de estudio [10].

Sin embargo, la investigación y caracterización de estos microorganismos requiere redimensionar el uso de micro técnicas en función de los problemas técnicos y complicaciones que se producen en este tipo de investigaciones [11]. En este sentido, la aplicación de técnicas y métodos que faciliten el estudio de estos microorganismos de forma eficiente, se hace cada vez más relevante [12]. Por tal motivo, a través de esta investigación, se pretende lograr describir morfológicamente amebas testadas del genero Arcella sp, a partir del uso de múltiples técnicas que se espera que faciliten y redimensionen su estudio.

\section{Materiales y Métodos}

El estudio de microeucariotas de vida libre requiere un abordaje multidisciplinario, ya que la observación de los mismos en ambientes acuáticos o sistemas astáticos naturales (entendiendo estos últimos como pequeños sistemas de agua estancadas que permiten el crecimiento de microbiota asociada a ellos, como por ejemplo heliconias, bromelias, etc), a través de un microscopio óptico, muchas veces no llegan a ser suficientes para una buena descripción, identificación y posterior caracterización del sistema. En este sentido, se aplicaron diferentes técnicas, tales como medios de cultivos, microscopía y morfometría, las cuales permitieron el estudio de estos microorganismos de forma eficiente.

Medio de cultivo: Para observar las características morfológicas de estos microorganismos, fue necesario cultivarlos en condiciones de laboratorio. Para ello, se tomó una muestra de $5 \mathrm{ml}$ de agua proveniente de sistemas astáticos, la cual fue sembrada en un medio de cultivo salino previamente estandarizado a $\mathrm{pH} 7$ y temperatura cercana al ambiente $\left(20^{\circ} \mathrm{C}-26^{\circ} \mathrm{C}\right)$.

Posteriormente, se monitoreó la abundancia de microorganismos colectando $5 \mu \mathrm{l} /$ medio y observándolo por técnicas de microscopía, dos veces por semana, durante un periodo de 4 semanas. Estos datos de abundancia,fueron reportados en concentración (organismos $/ \mathrm{ml}$ ).

Téenicas de Microscopía: Una vez alcanzado el punto de mayor abundancia total de microeucariotas de vida libre en los medios de cultivos utilizados (el cual fue de 3415 organismos $/ \mathrm{ml}$, de los cuales 1126 organismos $/ \mathrm{ml}$ correspondían al grupo de los ameboides), se procedió a realizar su observación a través de técnicas de microscopía, las cuales permitieron la descripción y posteriormente la identificación de estos microorganismos. Para esto, se siguió la metodología planteada previamente por Bracalé et al. [13].

Microscopía óptica: Para la observación de microorganismos por microscopía óptica (MO), se tomó una muestra de $5 \mu 1$ de medio, los cuales fueron colocados en un portamuestra de vidrio y observados de forma directa al microscopio óptico (marca Motic - BA310 Series), realizando el conteo de organismos vivos por campo observado, realizando un barrido total de 100 campos por muestra. Finalmnete, se realizó un registro fotografico con la camara principal de un Huawei Ascend Y200 de 3.1 megapixeles.

Microscopía Electrónica de Barrido: Para la observación de microorganismos de una muestra acuosa por microscopía electrónica de barrido (MEB), se realizó una estandarización del método convencional, utilizando una modalidad de barrido de bajo vacío o fuerza ambiental (ESEM), para la cual, se tomó una muestra de $5 \mu 1$ del medio de cultivo, la cual fue fijada con $5 \mu 1$ de gluteraldehído al $2 \%$ por 15 min y postfijada con $5 \mu l$ de tetraóxido de osmio al $1 \%$ por $15 \mathrm{~min}$. Una vez fijada y post-fijada la muestra, se tomaron $5 \mu$ de la mezcla y se colocaron en una rejilla de cobre cubierta previamente con una película de nitrocelulosa (colodión) y una película de carbono. Posteriormente, se colocaron las rejillas en una estufa a temperatura ambiente $\left(25-30^{\circ} \mathrm{C}\right)$ por $20 \mathrm{~min}$ para que la muestra se secara y posteriormente colocarla en el microscopio electrónico.

Finalmente, la muestra fue observada en un Microscopio electrónico de barrido (MEB), marca FEI Quanta 250 FEG, en modo de observación de vacío bajo y se realizó el registro fotográfico de las muestras

Análisis Morfométrico: A partir de las imágenes digitalizadas obtenidas previamente con ayuda de la técnica de microscopía electrónica de barrido (MEB), se obtuvieron las dimensiones de las características diagnósticas de interés de estos microorganismos, como lo son el ancho y el largo de la circunferencia de la célula y el ancho y largo de la circunferencia del pseudostoma. La toma de medidas 
fue realizada a través del programa ImageTool 3.0, con un posterior análisis de los datos a través de un paquete estadístico convencional.

\section{Resultados y Discusión}

Fueron descritos dos grupos de amebas testadas, de las cuales se plantea una posible identificación hasta el nivel de género con ayuda de microscopía óptica, microscopía electrónica de barrido y el uso de material bibliográfico pertinente [8], [14], [15].

El género descrito en este estudio fue Arcella sp., el cual es un grupo que presenta una teca circular que bordea al pseudostoma ubicado en la región oral (Figura 1), además de las características diagnósticas de cada grupo. Para el caso particular de las amebas de este estudio, las tecas observadas fueron de forma circular. No obstante, se pueden encontrar múltiples formas de teca: cilíndricas, ovales, con forma de estrella, circulares, entre otras, las cuales son características de cada grupo.

Con ayuda de la microscopia óptica, se cuantificó una abundancia promedio de $84\left({ }^{+} /-3\right)$ organismos $/ \mathrm{ml}$ a las cuatro (4) semanas de experimentación, cuando se reportaron los mayores valores de abundancia de la cinética de crecimiento.

En lo referente a la morfometría, se obtuvo un diámetro celular promedio de 59,07 $\pm 0,88 \mu \mathrm{m}$. Del mismo modo, se obtuvo el diámetro promedio del pseudostoma de 20,70 \pm $0,58 \mu \mathrm{m} y$, finalmente, se reportaron medidas de la membrana en la depresión del pseudostoma en la que se observó una red de celdas en formas regulares y geométricas, las cuales están conformadas por hexágonos o pentágonos, con una dimensión de lado promedio de $640 \pm 40 \mathrm{~nm}$.

\section{Técnicas de Microscopía}

Las amebas testadas del género Arcella sp. fueron observadas a traves de la técnica de microscopía optica convencional y por medio de ésta, se pudo describir su morfología básica e identificar algunas estructuras que son diagnósticas en la identificación de estos microeucariotas, como lo es su forma (Figura 1.A), la abertura del pseudostoma, la cual consiste en una abertura o boca circular ubicada en la region oral de la celula, la cual le permite a la ameba interactuar con su medio externo [7], así como la presencia de dos nucleos, como se aprecia en la Figura 1.B.

De igual forma, se apreciaron detalles de la teca, la cual por lo general, está compuesta de material secretado por el mismo microeucariota. Sin embargo, puede presentar sílice, granos de polen, hifas de hongos y otros detritus orgánicos presentes en el medio [16].

Para lograr una descripción y caracterización más eficiente, se incluye el uso de técnicas de microscopía de mayor poder de resolución como lo es la microscopía electrónica.

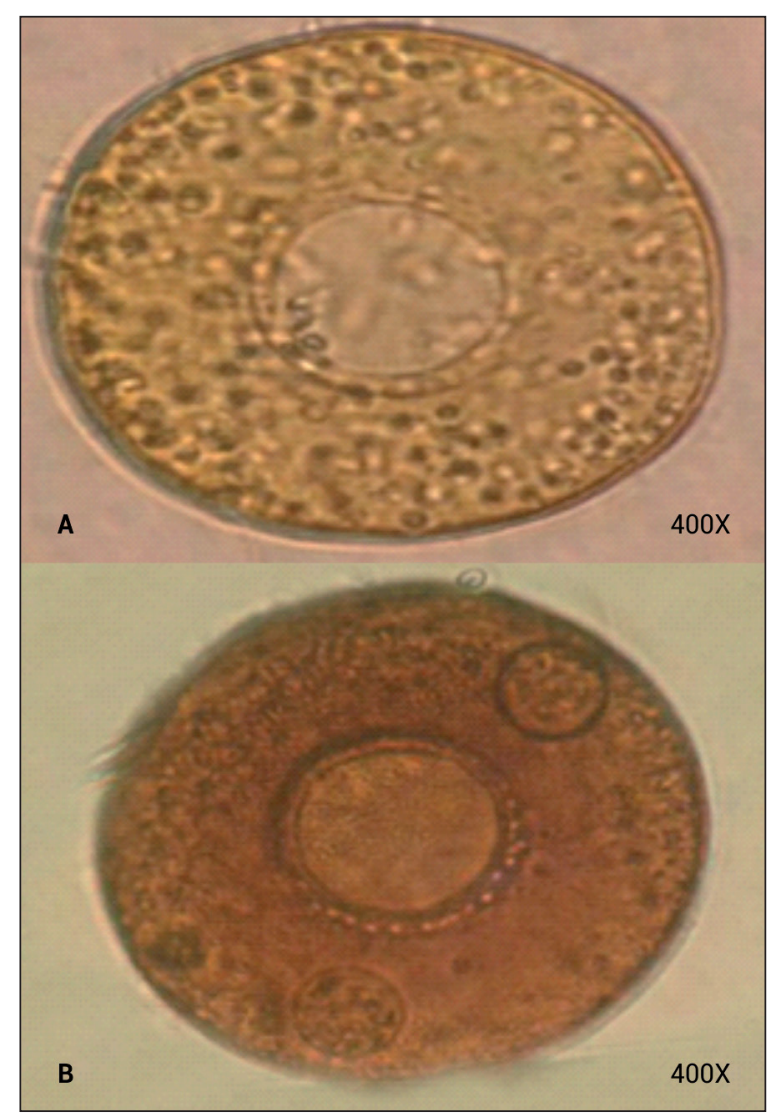

Figura 1: Fotografías de microscopia óptica de amebas testadas pertenecientes al género Arcella sp. A. Ameba testada, que presenta su abertura del pseudostoma. Se observan múltiples puntos en el interio de la célula, los cuales pueden corresponder con células bacterianas ingeridas por la ameba como alimento. B. Fotografías de microscopia óptica de una ameba testada, la cual presenta su pseudostoma en la region oral, así como dos núcleos que se ubican hacia extremos opuestos de la celula dentro de su citoplasma.

En este sentido, el microscopio electrónico de barrido (MEB) proporciona una profundidad de enfoque notable confiriéndole a las imágenes del microscopio de barrido su calidad tridimensional [17], lo cual es ideal para los estudios de morfo-funcionalidad e identificación de microorganismos presentes en micro comunidades, como las de este estudio.

En este sentido, la observación de estas amebas testadas por medio de técnicas de microscopía electrónica de barrido de bajo vacío, permitió profundizar en detalle aspectos de la morfología de estos microeucariotas, como la teca (Figuras 2 y 3), la abertura del pseudostoma (Figura 2.B y 3.A), los poros que la bordean y una red de celdas que recubre el interior del pseudostoma (Figura 3.A y 3.B). 

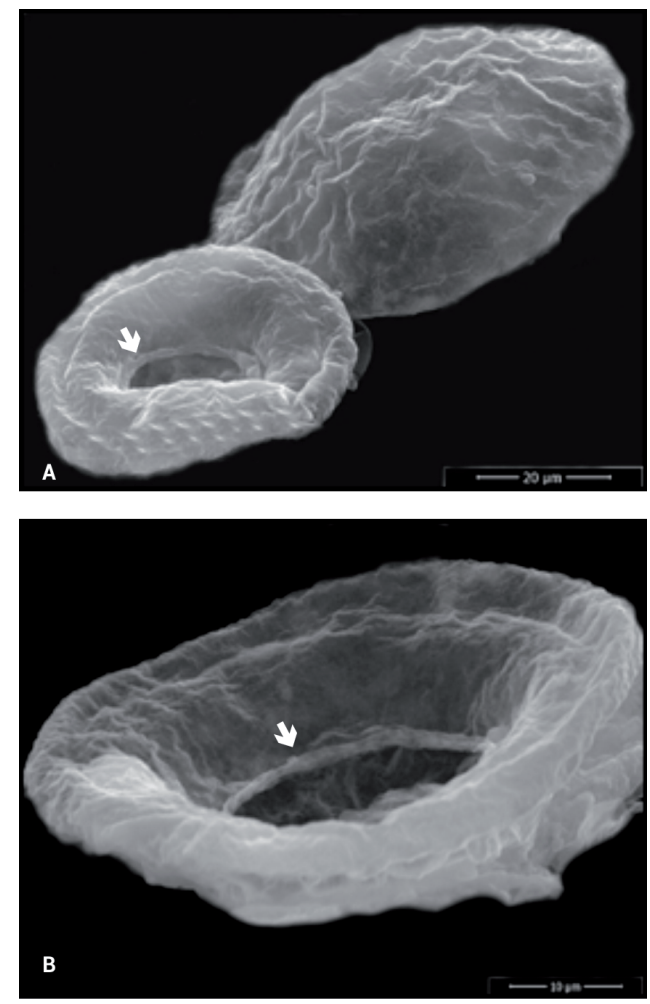

Figura 2: Micrografías de microscopia electrónica de amebas testadas del género Arcella.sp. A. Vista frontal y posterior de dos amebas. En la cara frontal se aprecia la depresión del pseudostoma (flecha), mientras que en la cara posterior se aprecia la membrana del ectoplasma.

B. Vista lateral de una ameba del género Arcella, en la que se aprecia la depresión del pseudostoma (flecha)

En la actualidad, pocos son los estudios que abordan la descripción, caracterización e identificación de este tipo de microrganismos en estos sistemas naturales.
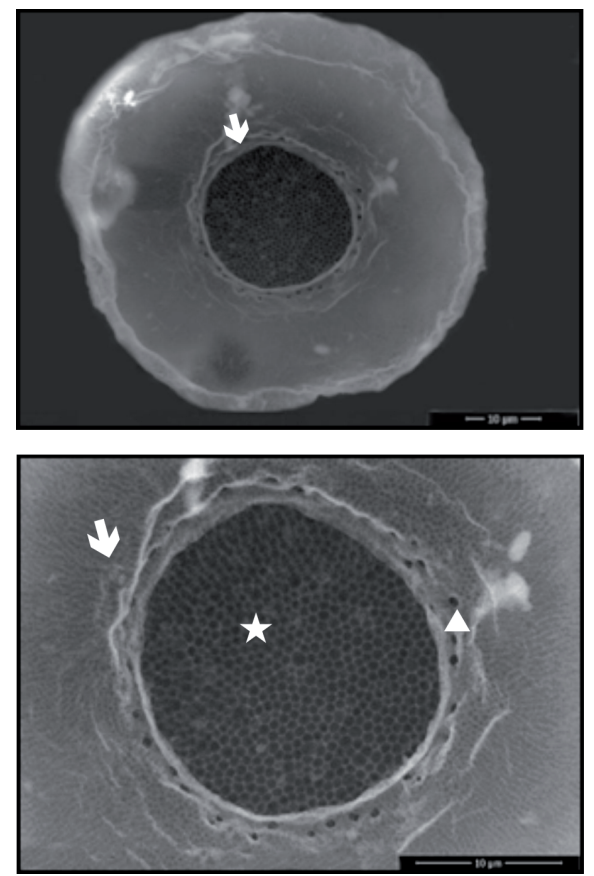

Figura 3: Micrografias de microscopia electrónica amebas testadas del género Arcella sp. A. Vista superior de un individuo del género Arcella en el que se aprecia la depresión del pseudostoma. (Flecha) y la teca redonda que recubre a la célula $\mathbf{B}$. Acercamiento de la zona oral que bordea al pseudostoma (flecha). De igual manera, se observa una red de celdas (estrella) que recubre el pseudostoma y los poros (punta de flecha) que se encuentran alrededor de la membrana de la abertura oral.
Es por esto que existe la necesidad de seguir ampliando los conocmientos en torno a estos microorganismos, ya que son grupos multidiversos y de elevada importancia para los ambientes bentónicos por su papel en los flujos de energías, las tramas tróficas y la conservacion de estos sistemas [2]. De igual manera, pueden ser utilizados como bioindicadores de contaminación ambiental.

Finalmente, es importante destacar que a partir de los resultados obtenidos en este estudio, por medio del uso de las técnicas de microscopía electrónica, y el uso de medidas morfométricas, las cuales fueron comparadas con referencia bibliográfica sobre el tema. Con base a los resultados obtenidos y a las referencias consultadas, los ejemplares estudiados pudieran corresponder con Arcella discoides, sin embargo, se recomienda complementar este estudio con el uso de técnicas moleculares que permitan corroborar la identidad taxonómica de estas amebas.

\section{Conclusiones}

La observación de muestras biológicas acuosas a través del microscopio electrónico en modalidad de bajo de bajo vacío y con la estandarización realizada a los métodos convencionales, resultó ser útil en la observación de estos microorganismos. No obstante, se recomiendan ensayos posteriores que permitan mejorar la calidad de observación de las mismas.

El estudio de la estructura y morfología de una comunidad de micro eucariotas de vida libre de un sistema natural se ve favorecido gracias al uso de múltiples técnicas que permitan abordar los problemas que puedan surgir del estudio de estos microorganismos, como los medios de cultivo y las técnicas de microscopía.

El nivel de resolución aportado por la técnica de microscopía electrónica en la identificación de características morfo-funcionales (diagnósticas), permitió describir morfológicamente amebas testadas, que partiendo de los resultados obtenidos y la comparación bibliográfica realizada, se sugiere la clasificación de Arcella sp para los microorganismos observados en este estudio. Sin embargo, se recomienda profundizar con métodos moleculares que permitan constatar la veracidad de estos resultados.

\section{Agradecimientos}

Centro de Microscopía Electrónica Dr. "Mitsuo Ogura" de la Facultad de Ciencias de la Universidad Central de Venezuela.

Dr. Ernesto González Profesor Titular de la Facultad de Ciencias de la UCV y director del Instituto de Biología Experimental (IBE). 


\section{Bibliografía}

1. Adl, S., Simpson, A., Lane, C., Lukes, J., Bass, D., Bowser, S., Brown, M., Burki, F., Dunthorn, M., Hampl, V., Heiss, A., Hoppenrath, M., Lara, E., Gall, L., Lynn, D., Mcmanus, H., Mitchell, E., Mozley-Stanridge, S., Parfrey, L., Pawlowski, J., Rueckert, S., Shadwick, L., Schoch, C., Smirnov, A., Spieget, F. 2012. The Revised Classification of Eukaryotes. J. Eukaryot Microbiol. 59(5): 429-493.

2. Neville, L. A., Christie, D. G., McCarthy, F. M. G., \& MacKinnon, M. D. 2010. Biogeographic variation in Thecamoebian (Testate amoeba) assemblages in lakes within various vegetation zones of Alberta, Canada. International Journal of Biodiversity and Conservation, 2, 215-224.

3. Medioli, F. S., \& Scott, D. B. 1988. Lacustrine thecamoebians (mainly arcellaceans) as potential tools for palaeolimnological interpretations. Palaeogeography, Palaeoclimatology, Palaeoecology, 62, 361-386.

4. Patterson, R. T., \& Kumar, A. 2002. A review of current testate rhizopod (thecamoebian) research in Canada. Palaeogeography, Palaeoclimatology, Palaeoecology, 180, 225-251.

5. Escobar, J., Martínez, J. I., \& Parra, L. N. 2005. Thecamoebians (Testaceous rhizopods) from a tropical lake: La Fe Reservoir, Antioquia, Colombia. Caldasia, 27, 293-298.

6. Van Hengstum, P. J., Reinhardt, E. G., Beddows, P. A., Huang, R. J., \& Gabriel, J. J. 2008. Thecamoebians (Testate amoebae) and foraminifera from three anchialine cenotes in Mexico: low salinity (1.5-4.5 psu) faunal transitions. Journal of Foraminiferal Research, 38, 305-317.

7. Blanco, M. 2003. Aspectos reproductivos y variaciones intraespecificas de amebas testáceas subtropicales y pampásicas. Universidad de La Plata. Buenos Aires - Argentina. Disponible en: http://hdl.handle. net/10915/4579.

8. Sigala, I., Lozano, S., Escobar, J., Pérez, L., Gallegos, E. 2015. Testate Amoebae (Amebozoa: Arcellinida) in Tropical Lakes of Central Mexico. Rev. Biol. Trop. 64 (1): 393-413.

9. Scorza, J. 1997. Introducción al conocimiento microscópico de ambientes astáticos. Centro “José W Torrealba”. Núcleo Universitario "Rafael Rangel" Trujillo - Venezuela.
10. Vaerewijck, M., y Houf, K. 2015. El papel de los protozoarios de vida libre transmitidos como patógenos en alimentos. Elsevier Ltd. Universidad de Gante. Bélgica.

11. Laybourn-Parry., J. 1984. A funtional Biology of Free-Living Protozoa. Berkeley and Los Angeles, California. University of California.

12. Ramírez, A., Chiou, W., Faber, K. 2000. Estudio Comparativo de Técnicas de Preparación de Muestras para Microscopía Electrónica de Transmisión de Recubrimientos Cerámicos Proyectados por Plasma. Bol. Soc. Esp. Cerám. Vidrio, 39(6): 735-740.

13. Bracale, K., Errico, L., y González, R. 2016. Microscopía Electrónica de Barrido de Presión Variable en la Identificación de Amebas Testadas del Genero Arcella sp. Acta Microscopica, Vol. 25 Supp. B., (2016). Presentado en el XVII Congreso Venezolano de Microscopía y Microanálisis (CONVEMI 2016). Caracas - Venezuela.

14. Larh, D. 2006. Taxonomía dos Arcellinida Kent, 1880 (Protista: ramicristates) do Parque Ecologico do Rio Tiete. Tesis de grado (Maestría). Instituto de Biociencias da Universidad de Sao Paulo. Departamento de Zoología. Sau Paulo - Brasil.

15. Junio, L., Fonseca, A., Mucio, G., Machado, L., Lansac-Toha, F. 2005. Composição de amebas testáceas (ProtozoaRhizopoda) de dois córregos do Estado de São Paulo, incluindo novos registros para o Brasil. Acta Sci. Biol. Maringá, 27(2): 113-118.

16. Charman, D.J., Hendon, D., Woodland, W. 2000. La identificación de tecamebas (Protozoa: Rhizopoda) en turbas. Guía Técnica de la Asociación de Investigación del Cuaternario. Asociación de Investigación del Cuaternario. Londres - Inglaterra.

17. Karp, G. 2009. Biología Celular y Molecular. Conceptos y Experimentos. McGraw-HILL Interamericana. 5ta edición.

Recibido: 25/10/2018.

Aprobado: 20/05/2019. 